\title{
Study on Demand Characteristics and Market Segmentation of Sweet Potatoes in Beijing Urban Area
}

\author{
Lixin Zhang1, a, Ruihan Liu', b and Siyu Lei ${ }^{3, c}$ \\ ${ }^{1,2}$ College of Economic \& Management, Beijing University of Agriculture and Beijing New Rural \\ Construction Research Base, Beijing,102206, China; \\ ${ }^{3}$ Agricultural Bank of China Beijing Branch Xuanwu Subbranch, Beijing, 100053, China; \\ a1075452156@qq.com, bruihanliu@163.com, ‘willsy0307@126.com
}

Keywords: Sweet potatoes, Demand characteristics, Market segmentation.

\begin{abstract}
We investigated the consumption characteristics of sweet potatoes in thousands of consumers made a detailed analysis of the market segmentation in this research. The result shows that the majority of consumers are satisfied with the quality of sweet potatoes products and focus on the therapeutic effect and nutritional value of them. In the choice of goods, outdoor dining consumers are tended to purple potatoes and home cooking consumers regard the product expediently to buy as the first choice. In terms of purchase place, most consumers is given priority to the farmer's market, community shop and the supermarket, however, the proportion of online shopping less than 3\%. By taking the consumer age, culture level, occupation and family year income as segmentation variables, the sweet potatoes consumption market is divided into two submarkets that is "core interests" and "functional extension". Consumer groups in the former market segments are more sensitive to the appearance, taste, price, and convenience of consumption process focusing on products' property of material needs than others. Consumer groups in the latter attach more significance to diet \& nutrition function, and further emphasize deeper spiritual needs such as environmental protection, vegetarian. Sweet potatoes-production operators in suburbs of Beijing are able to choose the target market suited for their own management ability, which are beneficial to enhance their own ability to response market demand and increase the operating earnings by the needs of different market segments. According to the consumption characteristics of diverse market segments, managers also could establish appropriate industrial objectives, and then improve the industry support policy, promoting the gradual optimization of sweet potatoes industry supply structure and the rising of the industrial competitiveness.
\end{abstract}

\section{Introduction}

On the demand side, with the continuous optimization of the Beijing consumer diet, sweet potatoes nutrition and health care function gradually by more and more consumer recognition, the status of the fresh sweet potatoes in the consumer structure of Beijing urban area is increasingly important, meanwhile, the total demand of fresh sweet potatoes in Beijing city market also showed steady rise trend. On the supply side, the consumption of sweet potatoes in urban areas is mostly produced outside Beijing, but the production scale of local sweet potatoes decreased from 5.07 hectares in 2011 to about 2.47 hectares in 2016. The supply gap of sweet potatoes products showed an increasing trend. In order to solve this kind of phenomenon, based on the demand orientation, this paper analyzes the characteristics of the demand and market segmentation of sweet potatoes in Beijing Market, which hope to make good suggestions for the local sweet potatoes producers who can give full play to the geographical advantages, optimize the planting structure and improve the yield of sweet potatoes planting and it has great significance for the government consummating preferential policies to promote the market competitiveness of Beijing sweet potatoes industry at a macro level. 


\section{ANALYSIS ON CONSUMPTION CHARACTERISTICS OF SWEET POTATOES IN BEIJING}

Research Background. The contents of the survey mainly include the situations about consumption of sweet potatoes at home or outdoors. In the respondents, there are many women and married people and most of interviewees' age between 25-55 years old. Education level is concentrated in the high school to undergraduate, enterprises and public institutions staff more, the majority of respondents' annual household income between 5-20 million.

Statistical description of consumption characteristics of sweet potatoes in Beijing. (1) Most consumers are satisfied with the quality of sweet potato and pay attention to its dietetic therapy and nutritional function, thus showing a stable preference for sweet potato consumption. The survey showed that nearly $90 \%$ of respondents expressed satisfaction with the overall quality of sweet potatoes or more satisfied. Similarly, nearly $90 \%$ of respondents approve of the sweet potato diet and health effects. More than half of the respondents' willingness to buy sweet potatoes changed little with the seasons and the consumers with the highest preference for sweet potatoes had no effect on the consumption of sweet potatoes.

(2) Residential is the main consumption place of sweet potato for urban consumers in Beijing. Sweet potatoes are one of the most popular foods for consumers whether at home or outdoors. Respondents in the survey of $2 / 3$ choose to consume sweet potatoes at home, $1 / 3$ choose to consume sweet potatoes at outside. The ratio is about $2: 1$.

(3) Consumers prefer to eat purple potatoes when they eat at outside, however, if they cook at home, they accept whatever they could buy in the market. Based on the survey of consumers' choice of different types of sweet potatoes, we found that in the dining out of the crowd, $81 \%$ of consumers prefer purple potatoes and in the cooking at home group, more than $78 \%$ of consumers choose common yellow heart or white heart sweet potatoes which can easy buy in the market. Obviously, the convenience of purchase plays an vital role in promoting the sales.

(4) Generally speaking, consumers are satisfied with the convenience of the purchase of sweet potatoes, while the farmers market is the main channel for it. In the convenience of the purchase of sweet potatoes channel evaluation, respectively, $43 \%$ of respondents believe that the purchase of sweet potatoes is more convenient, and the $41 \%$ consider that is very convenient, hence the convenience of the purchase of sweet potatoes can highly meet the demand for consumption. Among many choices made by consumers to purchase channels, farmers' markets are the most frequently selected, followed by community food stores and supermarkets. The selected frequency of wholesale markets, rural fairs and online purchases were all selected at low frequencies.

\section{STUDY ON THE MARKET SEGMENTATION CHARACTERISTICS}

In this paper, 11 initial variables are used to analyze the consumption preference of consumers in Beijing urban area, and then to describe the consumption effect of sweet potatoes. First of all, we use the Likert five scales to allow consumers to evaluate the importance of the 11 variables; the second step is factor analysis with the evaluation results, and finally through the cluster analysis, we do a segmentation of the sweet potatoes consumer market.

Statistical description and Test of variables. (1) Consumers value the nutritional and health functions, price, appearance and taste of sweet potatoes. In the evaluation of the importance of the 5 level, the higher the mean value, the higher the respondents' evaluation of the variable; The bigger the standard deviation is, the greater the difference of the evaluation of the variables. Fig. 2 shows that variables which consumers setting store by having no difference in recognition are the nutrition health function, price, appearance and taste. Followed by the purchase of convenience, habit, brand and buying as a present.

(2) In the reliability and validity test, we get the internal consistency of each measurement statement and construct validity of the questionnaires all within the scope of acceptance. Using Cronbach's Alpha coefficient as the reliability test standard, the value of SPSS calculated by 0.785 , indicating that the survey data is more dependent, and the internal consistency of the questionnaire 
is designed in a reasonable range. Exploratory factor analysis was used to test the validity of the questionnaire, which was used to test the validity of the questionnaire. The joint degree of the calculated variables is more than 0.620 , which indicates that the construct validity of the questionnaire is within acceptable limits.

Factor analysis and Market segmentation. (1) Factor analysis showed that the factors of "economic", "diet \& taste" are the most important factors in the consumption of sweet potatoes. First, Bartlett sphericity test is used to determine whether the scale is suitable for factor analysis. The use of SPSS extraction of principal components of the 11 variables, the KMO value is 0.762 , indicating that the original correlation is strong; the corresponding Sig. value is 0, which indicates that the correlation coefficient matrix has a significant difference with the unit matrix. It's suitable for factor analysis.

Using principal component analysis method of factor extraction and orthogonal maximum variance rotation, the results (Table 1) reflect that the 11 variables of the questionnaire were extracted by 4 factors, the extraction degree was higher, and the cumulative variance contribution rate was up to $78.95 \%$, which means that the 4 factors have $68.95 \%$ explanatory power to the original 11 variables. The results of factor interpretation are as follows:

1) "Economic" factor: the load of these three variables, price, promotion and the convenience, on the factor is higher, which concentrates on the basic demands of the convenience and economy in the consumption of sweet potatoes.

2) "Sense" factor: the two variables of the taste and appearance of the factors on the load are higher, which reflect the demand related to basic sensory attributes in sweet potatoes products.

Table 111 Rotation matrix of variables

\begin{tabular}{cllll}
\hline Variables & Factor 1 & Factor2 & Factor3 & Factor 4 \\
\hline Price & 0.812 & & & \\
Promotion & 0.635 & & & \\
Convenience & 0.701 & & & \\
Appearance & & 0.734 & & \\
Taste & & 0.772 & & \\
Lifestyle & & & 0.743 & \\
Habit & & & 0.791 & \\
Nutrition & & & 0.803 & \\
health & & & & 0.642 \\
Brand & & & & 0.613 \\
Packaging & & & & 0.605 \\
Present & & &
\end{tabular}

Date sources: application of SPSS computing

3)"Diet \& Taste" factor: in this factor, the load of these three variables is higher, namely nutrition health, consumer tastes and spiritual aspirations reflecting the needs of the consumer spiritual level. The original statements of the three variables are "diet and health care", such as weight loss or disease prevention, "taste and orientation of life and consumption" and "the spiritual needs of faith, environmental protection, and vegetarianism etc.". The diversification of the development and popularization of sweet potatoes diet health care function, cooking culture and advanced consumption concept, is conducive to tap the potential of the deeper level of additional value.

4)"Social" factor: brand, packaging and gift, the load of these three variables is higher, which reflects the multi-level consumer demand of consumers in the quality, credibility, packaging and communication. Rarely seen in the market are the brand and the appropriate packaging and processing of fresh sweet potatoes, especially gift boxes. It is an important approach to meet the consumer's communication function of sweet potatoes consumption in order to excavate the connotation and added value of sweet potatoes products.

To sum up, the consumption of fresh sweet potatoes in Beijing urban areas includes the basic demands of people's sweet potatoes, such as eating habits and solving the problem of food, but there 
is also a need for a higher level of diversity, such as taste, environmental protection, and communication.

Cluster analysis divides the market into two types: "core interests" and "functional extension". The four standardized factor values are saved as new variables, and the dynamic Kmeans algorithm is used to cluster the four factors to obtain the cluster centers of each of the two factors, and each of them represents a fine molecular market (Table 2).

Table 2 Cluster analysis of sweet potatoes consumption factors

\begin{tabular}{l|c|c}
\hline \multirow{2}{*}{ Factors } & \multicolumn{2}{|c}{ Clustering } \\
\cline { 2 - 3 } & 1 & 2 \\
\hline "Economic" factor & 0.24981 & -0.30991 \\
"Sense" factor & 0.30493 & -0.10132 \\
"Diet \& Taste" factor & -0.11205 & 0.49889 \\
"Social" factor & -0.33276 & 0.56419 \\
\hline
\end{tabular}

Date sources: application of SPSS computing

The variance analysis showed that the $\mathrm{F}$ value of each factor was greater than 0.8 , and the Sig.value was 0 , which indicated that the difference between the 2 market segments was significant in the 4 factors, and the results were effective. Combined with the characteristics of the sample and the results of the questionnaire survey, the two market segments were named "core interests" and "functional extension". The number of samples of two sub market segments accounted for $46 \%$ and $54 \%$ of the total sample, which shows that the functional extension of the sub market of high population size.

The interpretation of the two markets is as follows:

1)Core interest sub Market: consumers in such markets are more inclined to "Economic" factor and "Sense" factor. It is more sensitive to the price, appearance, taste and convenience of purchase, so people pay more attention to the basic function of the commodity. Overall, the result is a negative number, that is, the attention of the consumer groups to the "Diet \& Taste" factor and "Social" factor, explaining that the group had a low degree of attention to the health care function of sweet potatoes, but also lacked the identity of the brand, communication and other functions.

2)Functional extension sub Market: consumers in such markets are more inclined to "Diet \& Taste" factor and "Social" factor. In the consumption of sweet potatoes, it is more willing to choose the brand products and give them the spirit and communication function. These parts of the consumer groups take the additional value of food outside the sweet potatoes seriously.

\section{CONCLUSIONS AND IMPLICATIONS}

Main research conclusions. (1) The majority of consumers is satisfied with the quality of sweet potatoes products and focus on the therapeutic effect and nutritional value of them. In the choice of goods, outdoor dining for the purpose of consumers is tend to pay more attention to purple potatoes while home cooking consumers regard the product expediently to buy as the first choice.

(2) In terms of purchase place, most consumers are given priority to the farmer's market, community shop and the supermarket.

(3) On the basis of the factor analysis to the consumer age, culture degree, occupation and family income for market segmentation variables, the sweet potatoes consumption market is divided into "core interests" and " functional extension " two sub markets. In the "core interests" segments market of consumer groups with older age, low income and culture as the main feature, consumer focus on material demand and are sensitive to the price, appearance, taste and convenience and so on. In the "functional extension " segments market of consumer groups with the young, highly educated, enterprises stable occupation and higher income as the main feature, consumers pay more attention to diet and nutritional functions of products, and then emphasize the deeper spiritual needs such as the environmental protection and the vegetarian demand.

Main revelation and Significance. (1) Producers can choose the appropriate target market according to their own production conditions and operating capacity. Production managers who have ability to develop new products, open up new channels, expand the brand and promotion 
efforts can select the " functional extension " market as the main target market and their marketing strategy also needs to be matched with the characteristics of the target consumer groups, to give more value-added sweet potatoes products, to meet consumer spirit demands exactly. Otherwise, these producers can choose the " core interests " sub market as the main target market, paying attention to reduce the cost, pick out the high-quality potatoes tubers with good shape and high disease resistance in the production with the aim of producing fresh sweet potatoes who's the price can be received by low-income persons in a good shape, which meet the fundamental needs actively of older age, culture and low-income consumer groups.

(2) Government managers at all levels can refer to the characteristics of the sweet potatoes market in Beijing and increase policy support contrapuntally to the local sweet potatoes industry to guide the production operators focusing on the economic and cultural advantages of Beijing. Now it needs to be on what consumers want in the "functional extension" market, optimize the supply structure of sweet potatoes products, improve the market competitiveness of Beijing sweet potatoes industry, and ultimately promote the production operators to increase income.

\section{ACKNOWLEDGEMENTS}

As a communication author, Ruihan Liu and the other authors wish to acknowledge financial support from Beijing Agriculture Innovation Consortium of Grain Industry (BAIC09-2018).

\section{REFERENCE}

[1] Smith,Wendll.(1956).Product Differentiation and Market Segmentation as Alternative Marketing Strategies[M].Journal of Marketing,21(July 1956):3-8

[2] Tony Lunn. (1986). Segmenting and constructing markets[A]. Robert M.Worcester and John Dow $\mathrm{n}$ ham eds. Consumer Market Research Handbook, Third Revised and Enlarged Edition[C].Elsevier Science Publisher.B.V.1986,387-423

[3] YoramWind. (1978). Issues and Advances in Segmentation Research[J]. Journal of Marketing Research, August1978,317-337.

[4] Ma Hongzhong. Market segmentation and its application [J]. "Market Research" Special Issue 2004 Procter \& Gamble prize essays.

[5] Wang Xia.Research on market segmentation based on customer satisfaction and customer loyalty [J]. Nankai business review, 2005, 8(5):26-30.

[6] Shi Youchun. A new paradigm of market segmentation: An Empirical Study Based on two different products [J]. Nankai business review, 2010, (3).

[7] Luo Jining. Review of market segmentation: Retrospect and Prospect [J]. Journal of Shandong University (PHILOSOPHY AND SOCIAL SCIENCES EDITION),2003,6:44-48. 